\title{
ANÁLISIS JURÍDICO DESCRIPTIVO DE LA VIOLENCIA FAMILIAR Y EL DAÑO A LA PERSONA EN EL DERECHO CIVIL PERUANO
}

\author{
DESCRIPTIVE LEGAL ANALYSIS OF FAMILY VIOLENCE AND DAMAGE TO PERSON IN \\ CIVIL PERUVIAN LAW
}

Verónica Cervantes S. ${ }^{1}$

Universidad Nacional de San Agustín de Arequipa, Perú

(RECIBIDO EL 18/01/2010, ACEPTADO EL 08/06/2010)

\begin{abstract}
RESUMEN
Se realizó una investigación descriptiva donde se analizaron las variables de violencia familiar y el daño a la persona desde un enfoque interdisciplinario de la psicología y el derecho. Se enfatiza la trascendencia de la violencia psicológica, el daño al proyecto de vida, y su repercusión en el curso de la existencia y en la realización del ser humano.

Se ha considerado que la violencia familiar es una forma de relación disfuncional en la familia que causa daño a la persona. Existe una elevada prevalencia de denuncias presentadas ante el Ministerio Público, hacia el futuro probablemente continúe incrementándose, lo que requiere reflexión y plantear soluciones.

Se cuestiona la legitimidad activa de la persona que sufrió daño porque falta la evidencia visible del daño sufrido. Es así como la psicología clínica puede demostrar, a través de técnicas e instrumentos, la relación de causalidad entre el evento ocurrido y el daño ocasionado.

El TEPT es el cuadro clínico que mejor describe a las víctimas de violencia familiar, se enfatizan los síntomas de reexperimentación e hiperactivación.
\end{abstract}

Palabras clave: Violencia familiar; daño; proyecto existencial; legitimidad activa; prueba del daño; TEPT.

\begin{abstract}
A descriptive study was conducted between family violence and damage of person as variables, from psychology and law interdisciplinary approach. On emphasizes psychological violence, damage to life project, its impact in human being existence and achievement.

Family violence is a type of dysfunctional relationship into family that causes damage to person. There is a high prevalence of reports to Public Department in Peru. Probably in the future it will continue increasing, and it is important to think carefully about it and find solutions.

We question the legitimacy to act to the person who suffered the damage, because there is a lack of the obvious evidence of suffered damage. So, through techniques and instruments

1 Psicóloga de la Universidad Nacional San Agustín de Arequipa.

E-mail: veronicamaria_ps@hotmail.com
\end{abstract}


from clinical psychology we can demonstrate connection of causation between happened event and caused damage.

The TEPT is the clinical syndrome that better describe victims from family violence. On emphasizes symptoms of experience back and hyperactivity.

Keywords: Family violence; damage; project life; legitimacy to act; test of damage; TEPT.

La persona es un bien jurídico protegido en el ordenamiento jurídico peruano, la protección comienza desde la concepción en el vientre materno. Los aspectos tutelados son: la vida, la integridad corporal, la psiquis, la identidad, la imagen, la honra, la privacidad, la vida en relación, la proyección futura, la libertad de conciencia y el trato igualitario.

El hombre, al ser libre, se vuelve un ente que valora, proyecta y crea. Y por ser temporal, es histórico, se despliega en el tiempo, se sustenta en su pasado para proyectar en el presente, su futuro. Asimismo, el ser humano tiene, aparte de una infinidad de deberes de los que derivan cada uno de sus derechos, un deber genérico consistente en "no dañar", el mismo que está presente en todos los derechos subjetivos.

Sin embargo, en nuestra sociedad podemos constatar el dramatismo que ha alcanzado la violencia familiar como un fenómeno que se va incrementando de forma alarmante y que afecta el desarrollo integral de las personas, específicamente el daño psicológico que sufren las víctimas y la frustración de su proyecto existencial de vida, configurándose de este modo el daño a la persona.

De acuerdo a los antecedentes investigativos existen estudios que han tratado los temas de violencia familiar y daño a la persona en forma independiente o aislada, desde distintas perspectivas. En la presente investigación se han combinado estas dos variables en un mismo estudio, considerando el enfoque interdisciplinario de la psicología y el derecho.

Teniendo en cuenta lo mencionado, los objetivos de la presente investigación pretenden esclarecer, en primer lugar, cuál es la trascendencia y la dimensión del daño a la persona en víctimas de violencia familiar y cómo a través de la psicología clínica se puede probar el daño a la persona.

Esta investigación es de tipo descriptivo y hemos aplicado el método objetivo del derecho positivo.

\section{VARIABLES BAJO ESTUDIO}

\section{El daño a la persona}

Según Fernández Sessarego (1996), el daño a la persona "significa el agravio o lesión a un derecho, a un bien o un interés de la persona en cuanto tal", comprendiéndose dentro de él "hasta la frustración del proyecto existencial de la persona humana".

El daño al proyecto de vida es el daño que compromete el ejercicio de la libertad y su exteriorización fenoménica, por lo que lesiona, destruye, hace imposible, total o 
parcialmente, la realización de nuestro proyecto de vida. Si bien somos libres para proyectar nuestra vida, la ejecución de nuestro proyecto existencial puede frustrarse por una acción dañina.

\section{La violencia familiar}

Se puede definir la violencia familiar como "una forma de relación o interacción disfuncional en la familia que causa daño a la persona. Se caracteriza por la existencia de una desigualdad de poder entre sus miembros, donde el que tiene el poder lo usa en forma irracional mediante acciones u omisiones físicas, psíquicas y/o emocionales que se dan en forma crónica, permanente o periódica. Lo que altera el equilibrio y armonía de la familia y perjudica el bienestar, la integridad física y psicológica; la dignidad, la libertad y el derecho al pleno desarrollo de los miembros de la familia."

\section{La violencia familiar y el daño a la persona}

El proyecto de vida que el ser humano se propone a diario discurrir es aquel que tiene que ver con el destino mismo de la persona. En él se juega el futuro, la realización plena, de acuerdo a la personal vocación. Tanto el daño biológico como el daño a la salud son, sin duda alguna, daños gravísimos que se pueden causar a una persona, ya que sus consecuencias pueden repercutir en el curso de su existencia y son más graves aun cuando se encuentran calificados por el vínculo familiar. Pero el daño más grande que puede ocasionarse es aquel que incide en el proyecto de vida. El truncamiento o frustración del mismo tiene radicales consecuencias y repercute en el curso de la existencia. Dañar el proyecto de vida es dañar la esencia misma del hombre.

\section{La prueba del daño a la persona}

Respecto de la prueba del daño existe consenso en la doctrina que la víctima debe demostrar la existencia del perjuicio del cual pide indemnización. La actividad del juez está destinada a fijar la existencia y la entidad cuantitativa del daño resarcible, pero esta actividad está subordinada a la suministración de prueba de parte de la víctima. La prueba debe dirigirse a demostrar la responsabilidad del obligado. Así que el punto de partida para demostrar la existencia del daño es la prueba de la relación de causalidad, es decir, la conexión necesaria entre el acto antijurídico y el daño.

\section{La legitimidad para obrar en el daño a la persona}

La legitimidad para obrar constituye un presupuesto de la validez de la relación jurídica procesal. Aunque es un concepto procesal, la legitimidad está referida a la pretensión y al objeto del proceso, esto es, al derecho sustancial reclamado

Los hechos fundantes del razonamiento del juez, que le conducen a condenar por daño a la persona o, a la inversa, que le inducen a rechazar la demanda por tal concepto, requieren 
prueba. Es posible que, en el hecho, tratándose de padres, hijos y cónyuge, la prueba resulte más fácil. Pero, técnicamente, no puede faltar y simplemente presumirse por el juez.

Existe otra circunstancia en la cual se hace también difícil encontrar dicho nexo causal. Existen situaciones en las cuales es posible que los eventos o estímulos que se asumen como determinantes del disturbio o desequilibrio psíquico o psicosomático hayan sido tan solo elementos desencadenantes, pero no la causa necesaria y suficiente de las consecuencias que se advierten.

\section{La relación de causalidad}

En atención a la apreciación de la relación causal es posible valorizar como elementos a tener en cuenta, la existencia, por ejemplo, de una "conducta concurrente" por parte de la víctima, o la existencia de alguna "predisposición" en la misma. La predisposición debe probarse por quien la alega. Como es obvio, solo se responderá por los daños que se causan, pero no por los preexistentes, y solo en la diferencia es que se justificará un derecho al resarcimiento. En estos casos, por tanto, deberá recurrirse al criterio de la proporcionalidad, es decir, a quien causa el perjuicio debe atribuirse la obligación de resarcir la diferencia, pero no la resarcibilidad del daño preexistente.

\section{La pericia psicológica del daño a la persona}

Corresponde a los psicólogos y psiquiatras evaluar tanto la gravedad o dimensión de la lesión psicofísica, como sus consecuencias patológicas. Al respecto, Milmaiene apunta que los expertos deben proporcionar al juez un claro perfil psicopatológico de la persona víctima del daño, para lo cual se ha de formular un previo diagnóstico estructural de la personalidad afectada, indicando luego el tipo de patología que ha desarrollado el agraviado (Fernández, C., 1996).

\section{El trastorno de estrés postraumático (TEPT) en víctimas de violencia familiar}

La violencia familiar se ajusta a la definición que el DSM-IV realiza de un acontecimiento traumático, esto es, que represente una amenaza para la integridad de la persona y que la reacción emocional experimentada implique una respuesta intensa de miedo, horror o indefensión (APA, 1994, 2000). Además, en la situación de maltrato doméstico están presentes algunos factores que suelen ser predictores de este cuadro clínico: la duración prolongada del trauma, las graves lesiones que provoca en quienes lo sufren o el bajo apoyo social que con frecuencia refieren las víctimas, entre otros.

Recientemente se han comenzado a llevar a cabo estudios para analizar el perfil de este cuadro clínico en víctimas de violencia doméstica que señalan que son los síntomas de reexperimentación e hiperactivación los más característicos del TEPT en estas mujeres. En concreto, serían la presencia de recuerdos desagradables y recurrentes sobre el maltrato, el malestar psicológico intenso al recordar el suceso, la dificultad para conciliar el sueño, los problemas de concentración y el estado permanente de hipervigilancia, los más frecuentes. 
A la fecha, los resultados obtenidos sobre factores predictores del TEPT no son del todo concluyentes. En relación con la violencia, parecen ser el maltrato psicológico, la severidad y frecuencia de los episodios violentos, la cercanía en el tiempo de la última agresión, y el haber sido forzadao a establecer relaciones sexuales, los predictores más fuertes de la respuesta postraumática. La falta de apoyo social y familiar es también un factor significativo en el desarrollo de este cuadro clínico.

\section{MÉTODO}

Se utilizó el diseño descriptivo. El estudio se hizo en base a la recopilación de las estadísticas por denuncias de violencia familiar ante el Ministerio Público desde el año 2004 al 2008 para determinar la dimensión del fenómeno en estudio y su proyección hacia el futuro.

La recopilación de información documental y el análisis de la misma nos ha llevado a determinar la trascendencia de la violencia familiar.

\section{RESULTADOS}

En el Perú, de acuerdo a los anuarios estadísticos que presenta el Ministerio Público de la Nación, se puede observar el notable incremento del número de denuncias en estos últimos años. Así, podemos apreciar según la Figura 2 que a partir del año 2005 las cifras comenzaron a ascender, de tal forma que hacia el año 2006, el número de denuncias aumenta en un $15 \%$ respecto al año 2005; en el 2007, el número de denuncias se incrementa en un $4.3 \%$ respecto al año 2006, y hacia el año 2008, el número de denuncias aumenta en un $2.5 \%$ respecto al año 2007.

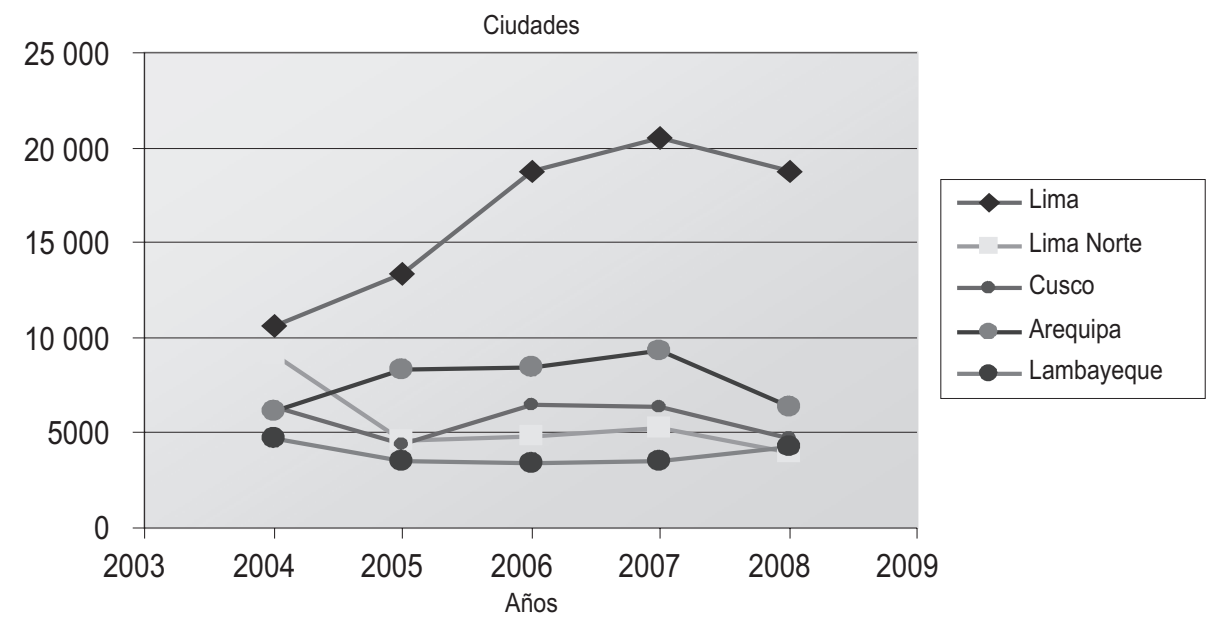

Figura $\mathbf{N}^{\circ}$ 1. Proyección anual de las denuncias por violencia familiar. 


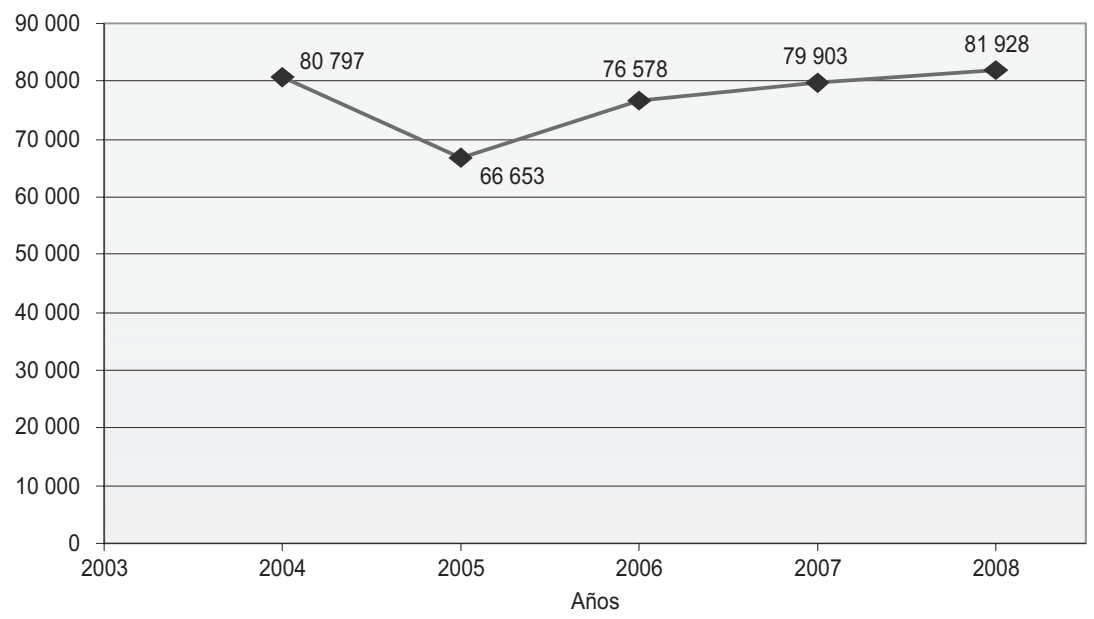

Figura N. ${ }^{2}$ 2. Proyección anual de las denuncias por violencia familiar en el Perú.

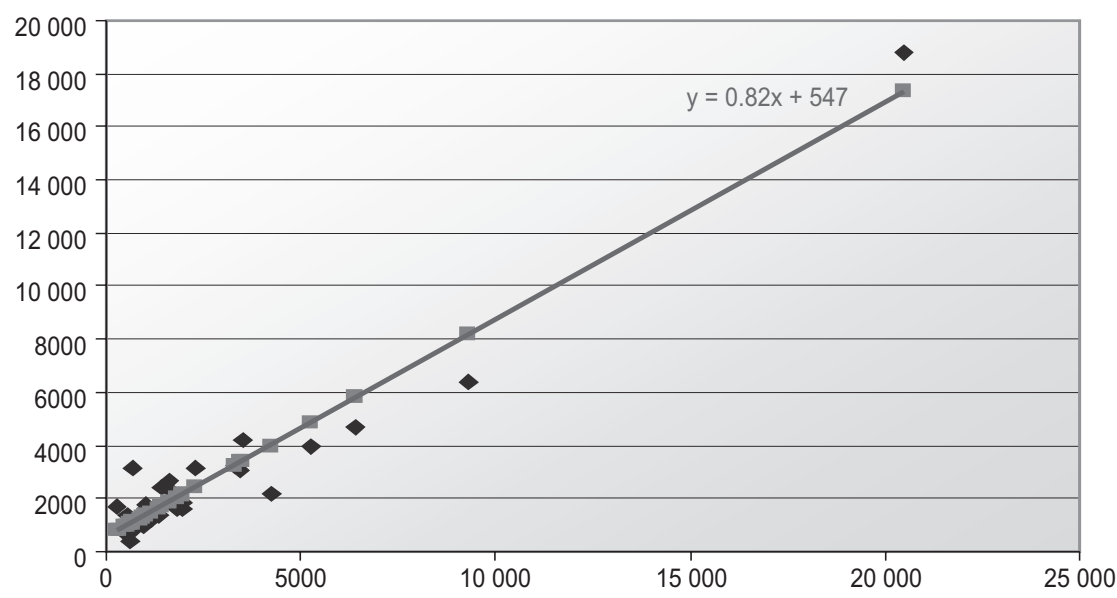

Figura N. ${ }^{\circ}$ 3. Diagrama de regresión.

Además de Lima, se puede observar que las ciudades con mayor índice de violencia familiar en estos últimos años son Arequipa, Cusco, Lima Norte y, recientemente, Lambayeque según datos de 2008 (ver Figura 1). En estas ciudades el número de casos de denuncias por violencia familiar ha ido cambiando, pero a diferencia de Lima las cifras de un año a otro no han variado significativamente y mantienen un promedio aproximado. Así, Arequipa presenta un promedio aproximado de 7705 denuncias por año; Cusco, 5682 denuncias por año; Lima Norte, 4676 denuncias anuales, y Lambayeque, 3864 denuncias por año.

Si hacemos un análisis del fenómeno de la violencia familiar en los últimos tiempos, en esta época contemporánea podemos darnos cuenta que el incremento sistemático en el número de denuncias interpuestas no necesariamente es el resultado de un aumento real 
de la violencia en el ámbito doméstico. Se ha señalado que este incremento podría ser un indicador del reciente despliegue en nuestra sociedad de recursos sociales a disposición de las mujeres maltratadas y de la mayor sensibilidad social ante el problema que favorece que las mujeres estén mejor informadas, se sientan más respaldadas y empiecen a cambiar la resignación por el ejercicio de sus derechos.

De manera contraria, el aumento del número de denuncias también se podría interpretar como un aumento real de la violencia familiar en el Perú durante los últimos años, como reacción al progreso de la situación social de la mujer y a la ruptura de los privilegios de poder masculino. Sin embargo, no hay datos disponibles que permitan valorar si esta interpretación es adecuada. Algunos datos podrían ayudar a defender más una interpretación que otra. Por ejemplo, el hecho que se hayan incrementado fundamentalmente las denuncias por malos tratos psicológicos, que son más difíciles de identificar, parece defender la idea de que el aumento de las cifras es el resultado de una mayor información y concientización social del problema.

Hacia el 2009, de acuerdo al diagrama de regresión de la Figura 3, se puede apreciar que las denuncias por violencia familiar seguirán aumentando en el Perú, lo que requiere plantear soluciones realmente efectivas, y tomar medidas acertadas y oportunas para que este fenómeno social se detenga, gracias a la intervención de los organismos de justicia implicados, a la participación de la comunidad en general y a la actuación de las víctimas en defensa de sus derechos y dignidad personal.

\section{DISCUSIÓN}

Si hacemos un análisis de antecedentes históricos, observaremos que en pleno siglo XX, bajo la influencia de la filosofía de la existencia y del personalismo jurídico, se comenzó a desarrollar un nuevo enfoque de los denominados derechos de la personalidad, a través del cual comienza una verdadera época de revisión de lo que es en realidad la persona. Se destaca el derecho a la vida, derecho a la salud, a la privacidad, al honor, a la imagen, al nombre y a la identidad. Por lo tanto, todo derecho fundamental menoscabado implica un daño a la persona; el cual incide en cualquier aspecto de la personalidad del ser humano, y por ello se le designa también como "daño a la integridad psicosomática".

El daño psicosomático puede recaer directamente en el cuerpo o soma del sujeto o en la psique, bajo el entendido que en cualquier caso el daño a una de tales esferas repercute, en alguna medida, en la otra sobre la base de la inescindible unidad que ambos constituyen.

Sin embargo, si bien las secuelas provocadas por el maltrato físico son más evidentes, el impacto a nivel psicológico y el deterioro en la calidad de vida de las víctimas por violencia familiar es más difícil de identificar y evaluar.

Una lesión psicosomática que incide preferentemente en la dimensión psicológica resulta, como es obvio, menos elocuente y visible, y probablemente más difícil de diagnosticar. $\mathrm{Y}$, en ciertos casos, puede pasar inadvertida para cualquier persona que no sea especialista en la materia. La lesión que afecta lo psíquico, en cualquiera de sus manifestaciones, puede ser el resultado de una previa agresión somática, aunque puede también presentarse inicialmente desvinculada de dicha agresión. 
El daño psíquico consiste en la existencia de alteraciones de la personalidad, se manifiesta a través de perturbaciones de la vida interior del sujeto y de las relaciones interpersonales y sociales, que cambian estable y sensiblemente la experiencia vital de la personalidad.

Fernández Sessarego (1996) precisa que el daño psíquico consiste, en cuanto lesión considerada en sí misma, en una alteración, modificación, perturbación o menoscabo, de carácter patológico del equilibrio mental del sujeto, generalmente permanente y de reconocida magnitud. Las connotaciones patológicas del daño psíquico pueden ser cuantificadas por los expertos, al igual que lo que acontece con el daño somático.

A su vez, todo derecho fundamental menoscabado implica un daño a la persona; el cual, cabe ser sistematizado teniendo en consideración la especial naturaleza bidimensional del ser humano. En tal sentido, el daño psicosomático y daño a la libertad o proyecto de vida constituyen una unidad sustentada en la libertad, núcleo de la existencia de los individuos.

El daño al ser humano, en su más honda acepción, es aquel que tiene como consecuencia la frustración del proyecto de vida de la persona, es un hecho de tal magnitud que truncaría la realización de la persona humana. Dañar el proyecto de vida es dañar la esencia misma del hombre.

El daño al proyecto de vida es el daño que compromete el ejercicio de la libertad y su exteriorización fenoménica, por lo que lesiona, destruye y hace imposible, total o parcialmente, la realización de nuestro proyecto de vida. Si bien somos libres para proyectar nuestra vida, la ejecución de nuestro proyecto existencial puede frustrarse por una acción dañina. El daño al ejercicio de este singular "proyecto de vida" frustra nuestro futuro, trunca el sentido de nuestra vida y crea un "vacío existencial" difícil de suplir.

Una de las manifestaciones más graves de la violencia es aquella que se presenta en el medio familiar; la que más daño puede causar al individuo, a la familia y a la sociedad, y es la que con más frecuencia actúa en nuestra vida cotidiana, constituyéndose en una parte fundamental del patrón de convivencia y cultura humanas.

Es importante resaltar que cuando se habla de violencia, se minimiza la que se ejerce en el entorno doméstico o familiar porque se ha "naturalizado" este tipo de relación, ya que al aceptarse que la violencia es una forma natural de comportarse y relacionarse, ha sido un factor influyente para su ocurrencia en nuestras sociedades.

La violencia familiar constituye un fenómeno que atenta indiscriminadamente contra la población femenina, convirtiéndose hoy en día en la principal amenaza para su integridad física y psicológica. El elevado impacto sobre la salud de quienes la sufren ha obligado a considerar esta forma de violencia como uno de los mayores asuntos de salud pública y de la defensa de los derechos humanos (Organización Mundial de la Salud, 2000).

La violencia familiar y, en general toda forma de violencia contra la mujer, es un problema muy extendido en el Perú. Según un estudio de la Organización Mundial de la Salud (OMS, 2002), en diez países del mundo, el Perú resultó ser el más violento contra la mujer. 
Ahora, para demostrar la existencia del daño a la persona en casos de violencia familiar debe existir una relación de causalidad entre el comportamiento del sujeto y la existencia del perjuicio, es decir, la conexión necesaria entre el acto antijurídico y el daño. Pero además, en lo que se refiere a los efectos, esta relación causal toma otra dimensión pues va a determinar la extensión del perjuicio.

La proyección de la relación causal de la existencia y entidad del perjuicio debe probarse. Por esta razón, en este tema adquiere particular importancia la realización de pericias y los análisis de las historias clínicas de las personas afectadas.

Para probar un daño subjetivo, inherente a la persona, debe acreditarse lo siguiente:

a) Que se tiene legitimación activa para demandar por daños;

b) El demandante de daño a la persona deberá acreditar, además, lo siguiente: que ha existido una agresión a su persona o a un bien o derecho de la personalidad, que en este caso se precisará nombrándolo. Aquí está contemplado el señalamiento concreto del bien jurídico protegido.

La legitimidad para obrar constituye un presupuesto de la validez de la relación jurídica procesal. Aunque es un concepto procesal, la legitimidad está referida a la pretensión y al objeto del proceso, esto es, al derecho sustancial reclamado.

La violencia familiar se ajusta a la definición que el Manual diagnóstico y estadístico de los trastornos mentales (DSM-IV) realiza de un acontecimiento traumático, esto es, que represente una amenaza para la integridad de la persona y que la reacción emocional experimentada implique una respuesta intensa de miedo, horror o indefensión (APA, 1994, 2000). Además, en la situación de maltrato doméstico están presentes algunos factores que suelen ser predictores de este cuadro clínico: la duración prolongada del trauma, las graves lesiones que provoca en quienes lo sufren o el bajo apoyo social que con frecuencia refieren las víctimas, entre otros. Todo ello nos conduce a la psicopatología del trastorno de estrés postraumático (TEPT).

Recientemente se han comenzado a llevar a cabo estudios para analizar el perfil de este cuadro clínico en víctimas de violencia doméstica que señalan que son los síntomas de reexperimentación e hiperactivación los más característicos del TEPT en estas mujeres. En concreto, serían la presencia de recuerdos desagradables y recurrentes sobre el maltrato, el malestar psicológico intenso al recordar el suceso, la dificultad para conciliar el sueño, los problemas de concentración y el estado permanente de hipervigilancia, los más frecuentes.

La revisión de estudios evidencia la alta prevalencia del TEPT en víctimas de violencia familiar, lo que permite concluir que, si bien esta categoría diagnóstica no es la única que presentan las mujeres maltratadas, sí sería la más apropiada para describir las repercusiones que a nivel psicológico provoca el maltrato.

Para probar la existencia de daño a la persona, la psicología clínica ofrece un respaldo científico para establecer la relación de causalidad y la legitimidad para obrar de quien dice ser víctima de violencia familiar, a través de los aportes científicos en el estudio de la personalidad normal y anormal, así como en el manejo de técnicas e instrumentos en la evaluación y diagnóstico de las enfermedades mentales. 


\section{CONCLUSIONES}

1. La violencia psicológica afecta la integridad emocional de las personas por la naturaleza del trauma o secuela psíquica que deja en la víctima, anulando de esta manera su proyecto existencial de vida y configurándose de este modo el daño a la persona.

2. La regulación de la violencia psicológica en el Derecho Civil peruano es deficiente, dado que no existe una medición clara y valoración del daño a persona, no se puede probar en forma idónea la magnitud del daño ya que el efecto incide en la esfera subjetiva de la persona, por lo que existe una tendencia a desvalorar los efectos perjudiciales de la violencia psicológica, lo que trae como consecuencia una desprotección de la víctima frente a esta.

3. Es posible determinar la relación de causalidad entre el daño a la persona y la violencia familiar a través de técnicas e instrumentos que ofrece la psicología clínica que permiten definir el perfil psicopatológico de la víctima, el diagnóstico de la personalidad, las predisposiciones preexistentes y la determinación de los síntomas del trastorno de estrés postraumático.

\section{REFERENCIAS BIBLIOGRÁFICAS}

1. Alonso, E. (2007). Mujeres víctimas de violencia doméstica con trastorno de estrés postraumático: validación empírica de un programa de tratamiento, (tesis de doctorado). Madrid: UCM.

2. Aquize, J. (2005). Tratamiento de la violencia familiar en la legislación peruana y comparada, (tesis de maestría). Perú: UCSM.

3. Junco, J. (2000). Violencia familiar en el Perú. En Revista peruana de psicología, $5,5,81-92$.

4. Fernández, C. (1996). Daño al proyecto de vida. En Revista de la facultad de derecho de la Pontificia Universidad Católica del Perú, 50.

5. Ordoqui, G. (1996). Pautas y criterios para la avaluación del daño a la persona. Montevideo: Del Foro.

6. Organización Mundial de la Salud (OMS) (2000). Violencia contra la mujer. Disponible en: http://www.who.int/inf-fs/en/fact239.html.

7. Rincón, P. P. (2001). Trastorno de estrés postraumático en víctimas de maltrato doméstico: evaluación de programas de intervención, (tesis de doctorado). Madrid: UCM.

8. Tamayo, J. (1990). De la responsabilidad civil. Bogotá: Themis.

9. Yucra, J. (2001). La violencia familiar en el ámbito de competencia de los juzgados especializados de familia, (tesis de maestría). Perú: UCSM. 